\title{
IoT and loE prototype for scalable infrastructures, architectures and platforms
}

\begin{abstract}
Internet of Things (IoT)is the third wave of economy after the first and second being agriculture and industry, respectively, paving the way for the fourth industrial revolution (4IR). IoT is a combination of all the revolutionary technologies in the last two centuries. More than a billion of smart devices have been developed across the world by more than 10 vendors to satisfy billions of needs that are trusted by $98 \%$ of economic actors. This study describes design and implementation of IoT architectures stressing on scalability, integration, and interoperability of heterogeneous IoT systems. It gives answers toi) how systems can be designed to become easily configurable and customizable for a specific IoT infrastructure? And ii) how Investors, producers and consumers can be integrated on the same page of an IoT platform? We have developed a master database and directories from top chart IoT nomenclature, frameworks, vendors, devices, platforms and architectures and gathered data from 27 big online resources commonly used by Forbes, Business week and CNBC. Also, datasheets of IoT equipment by vendors (e.g. Intel, IBM, ARM, Microchip, Schneider, and CISCO), used tools (e.g. Lab center Proteus, AutoCAD and Excel), and platforms (e.g. Visual Studio, Eclipse) are combined to build directories of plethora of data. The main outcome of this work culminates in providing a seamless solution and recommendations for various infrastructures (hardware and software), for effective and integrated resource utilization and management in an IoT perspective.
\end{abstract}

Keywords: internet of everything, web of things, social-internet of things, internet of things, infrastructure, architectures
Volume 4 Issue 5 - 2018

\author{
Farid Touati,' Hasan Tariq,' Mohammed A \\ Al-Hitmi,' Adel Ben Mnaouer, ${ }^{2}$ Anas Tahir,' \\ Damiano Crescini ${ }^{3}$ \\ 'Department of Electrical Engineering, Qatar University, Qatar \\ ${ }^{2}$ Canadian University of Dubai, UAE \\ ${ }^{3}$ University of Brescia, Italy
}

\begin{abstract}
Correspondence: Farid Touati, Department of Electrical Engineering, Qatar University, Qatar, Tel (974)4403-422I, Fax (974)4403-420I, Email touatef@qu.edu.qa
\end{abstract}

Received: September 10, 2018 | Published: October II, 2018

\section{Introduction}

By 2020, the number of connected machines is estimated to reach more than 34 billion-more than four folds the globe population. A smart IoT solution potentially delivers significant benefits such as reduced device support and maintenance costs, customer engagement and satisfaction, and new business models. Infrastructure ${ }^{1}$ ecology refers to the scalable internal or invisible relationships and functional mechanisms of elements in a particular system having dependencies to work as a single unit by best space utilization mechanisms of having vegetated/green roof tops and underground scalable construction for all type of architectures having habitats for terrestrial animal species as well, only lacked the facility management aspects. In a green and blue ecological metropolitan literature analysis by ${ }^{2,3}$ infrastructure refers to the entire set of systems and subsystems including transportation, water, energy, education, health, food, telecommunication, defense, buildings and industries that define eco-system of a region, country, state or city using data collected by different research portals. Recent IoT work ${ }^{4}$ is based on Urban Sense (DCU), Bus Net and its effective integration using Representational State Transfer (REST) Application Programmer Interface (API), European Telecommunications Standards Institute (ETSI) and FIWARE. Geo-mapping used in it is an excellent infrastructural tool that requires cost benchmark to be addressed and led to platform level development in terms of transportation routing protocols. ${ }^{4} \mathrm{On}$ the other hand, Government, public and private sector are three Internet of Everything (IoEs) ${ }^{5}$ that govern all the lower corporate and enterprise IoEs like defense, civil and commercial sector, which are in turn associated with trees of IoTs such as army, air force, navy, police, law, oil and gas, pharmaceutical, chemical andindustries. ${ }^{6-11} \mathrm{~A}$ an excellent analysis from Figures 1-9 and Table 1 comparison ${ }^{6}$ among open MTC, FIWARE, Site Where and Webinos, the proprietary AWS IoT, IBM's Watson IoT, Microsoft's Azure IoT Hub and Samsung's Smart Things platforms. Work by ${ }^{6}$ proved very fruitful for our work in time saving aspect decision making in development of our work. Lacked discrete recommendations and solution of their IoM (Integration of Middleware) analysis. Huge gaps in terms of authentication and identifications addressed by ${ }^{7}$ based on Xing QR need more elaboration with respect to multiple signatures as QR is not the only code being used, secondly for compression and smart character generation for scalability can handle billions of users. Interoperability and integration scenarios and methods proposed ${ }^{8,9}$ are need to improve in terms of real-world devices information. Excellent contribution in terms for IAM (Identity and Access Management) by Nitin Naik et al. ${ }^{14}$ in terms of comparison of SAML, OAuth, OpenID Connect \& SSO for mobile devices has been used by us in a different ubiquitous sense at D2D level. Physical hardware implementation and specifications of systems was the deficiency. ${ }^{14}$ HTTP 1.1 , HTTP2 and SPDY comparison as new alternatives by Nitin Naik ${ }^{15}$ reduced our effort in decision making. Contribution led us to better understating prospect ${ }^{16}$ Tables $1 \&$ Table 2 in terms of transmission and energy constraints of Message Queuing Telemetry Transport (MQTT), Constrained Application Protocol (CoAP), Advanced Message Queuing Protocol(AMQP), Extensible Messaging and Presence Protocol (XMPP), Transmission Control Protocol (TCP) and Web sockets on Intel and ARM Industry Standard Architectures(ISAs). Contribution [16] needs to be improved in the sense of verification on some ready to go IoT topology in GNS3, Wireshark and VMware based platforms. Hand-Off time calculations by Jorge $\mathrm{E}$ et al. ${ }^{17}$ prove AMQP better than MQTT for $0.5 \mathrm{~KB}$ to $1.2 \mathrm{~KB}$. Work needs to be matured for HTTP, CoAP and Web sockets. A very noticeable 
and referable effort by Peter Ruckebusch et al. ${ }^{18}$ for Tiny OS, RIOT and Contiki OS based embedded local and network control engine needs to address the effect of database integration used. Green aspect, energy harvesting and nano-power implementations by Farid Touati et al. ${ }^{19,20}$ and the SERENO module for air quality ${ }^{21,22}$ needs to be assessed for mass production from scalable aspects and digital infrastructure definitions, being an excellent novel contribution. ${ }^{23}$ Finally the glue. Things mash-up platform ${ }^{24}$ developed from inspirations WoT Kit, Paraimpu and servIoTicy. NODE-RED having its functions programming using JavaScript has a little effected its popularity and flexibility. Cloud virtualization design proposed by Ken J. Mbuki et al. ${ }^{25}$ for education and for community using by Weitao Xu et al. ${ }^{26}$ using thin clients had application limited as compared to capability of hardware. Web of things (WoT) has got a plenty of attention in market as per its flexibility and diversity. Private information sources of layman are now only three. ${ }^{27}$ This challenge increases in terms of involving SMS and email. The WoT has to be very structured and well defined in terms of segments to model any infrastructure first and then implement it. In our work, we have tried to fill the potential gaps and propose two scalable IoT models, namely Infrastructure-Centered Model and Agent-Centered Model. Key advantages of our designs and implemented systems are:
a. Infrastructure Definitions and Parametric Model
b. Device and Implementation Cost Scaling
c. IoT Integration of Middleware
d. Multiple Digital Signatures, Identification \& Authentication

e. Runtime Data Compression and Reduced Network Traffic

f. Systems \& Network Simulation and Design onboard

g. Multiple Data Sources SQL and non-SQL (SQlite, MySQL, PostgreSQL, noSQL, JSON, XML)

h. Utility Billing and Service Integrity

i. Multiple IoT Platforms

j. Multiple IoT Architecture and Hardware Options

k. Multiple OS and RTOS with Images available

1. Multiple Brokers and Open Source

m. Multiple Enabling Technologies (Wired, Wireless, Fiber and SAT)

n. Infrastructure Driven User Interface (UI) and User Experience(UX)

o. Multiple graphs, Geo-Plots and Comparison Options

p. Commercial grade hardware safety and operations

q. Financial Flexibility as per Infrastructures and Consumer Category

r. Life-Cycle Management with Garbage Collectors

s. Hardware Architecture Characterization and Classification

t. Time stamped SMS, Email and Social Media Posting
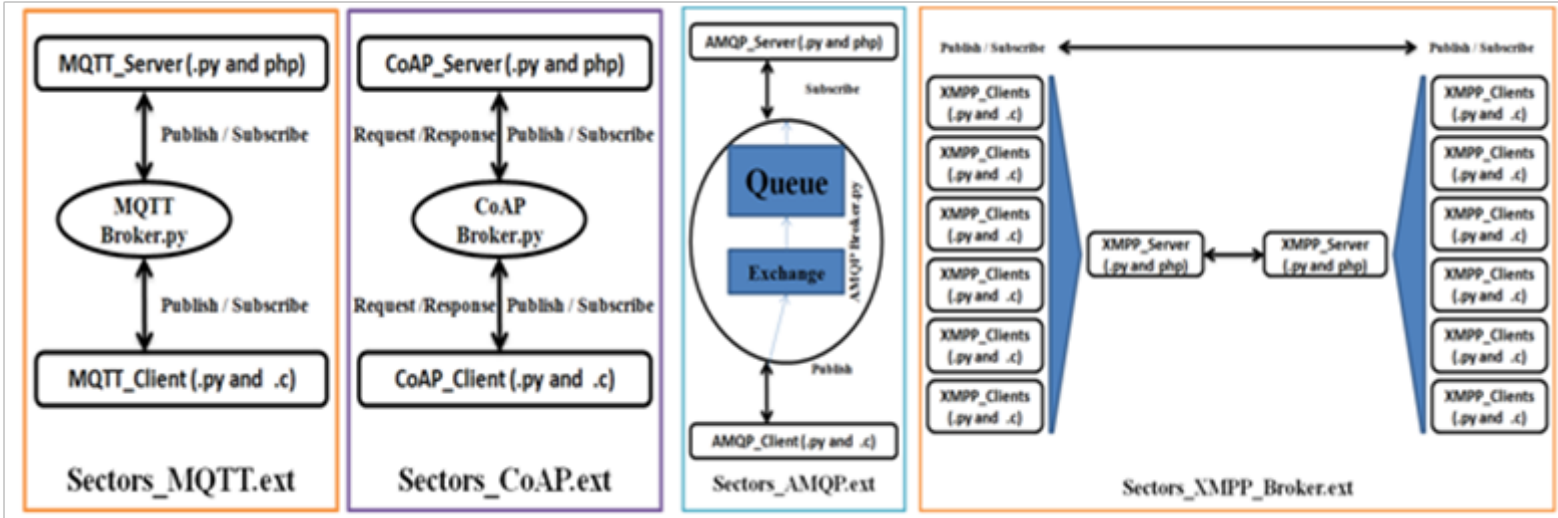

Figure I Multiple Brokers for Sectors Infrastructure

Table I loT/loE Packages Classification

\begin{tabular}{lllll}
\hline Parameters & Basic Packages & Value Package & Premium Package & Revision \\
\hline PS-PB & SC-37F-PDAB & SC-37F-PDAB & SC-37F-PDAB & X- 0 \\
PS-PV & $X$ & SC-35F-PDAB & SC-35F-PDAB & X-0 \\
PS-PP & $X$ & X & SC-68F-PDAB & X-0 \\
\hline
\end{tabular}

Table 2 DAB Tagging Schema

\begin{tabular}{lllll}
\hline Enclosures Size (inches) & Parameters & PCB Topology & Revision & \\
\hline$($ Length $\times$ Width $\times$ Depth $)$ & $(\mathrm{B}, \mathrm{V}$ and $\mathrm{P})$ & $($ No. of I/O Headers $)$ & $(\mathrm{X}-0)$ & \\
$3: 3 \times 3 \times 2$ & CurrentB & $4=\mathrm{F}$ for Basic & $\mathrm{A}$ & 0 \\
$6: 6 \times 3 \times 2$ & VoltageB & $7=\mathrm{S}$ for Value & $\mathrm{B}$ & $\mathrm{I}$ \\
$10: 10 \times 6 \times 2$ & FrequencyB & $9=\mathrm{N}$ for Premium & $\mathrm{C}$ & 2 \\
$14: 14 \times 10 \times 6$ & Digital LockB & & $\mathrm{D}$ & 3 \\
\hline
\end{tabular}


Our IoT system design and implementation offers financial scalability in three levels of parameters called parameters sets (PS) where (PB: population basic, PV: population value and PP: population premium) based on sensor cards (SC-SXT-CATX) where (S: size of enclosure, $\mathrm{X}$ : number of parameters and T: PCB topology and CAT: category). Revision has two parts (X: set of alphabets and 0: sub revision, set of natural numbers). See Table 1, for details see Table 2 . Parameters, sensors, enclosure sizes, PCB topologies and categories are given in later sections. Ensuring availability of smart devices (IoT) everywhere is the key motive of this work to bring technology to the purchasing power level of everyone. Basic package contains utility sensors for billing and security, value package contains indoor air quality and premium value contains structural health monitoring, environmental and early warning sensors.

\section{Scalable and interoperable models}

\section{Infrastructure-centered model (ICM)}

MQTT, CoAP, AMQP and XMPP multiple brokers for IoE and IoT, OPC UA for Industrial IoT(IIoT) and one block using DDS and Web sockets is our contribution to ensure QoS and interoperability at the same time. Our contribution facilitates all the cases for M2M, D2D, M2C, M2D, C2C, D2S, M2S, S2C, S2S (M: machine, D: device, S: Server and C: cloud). Information model for hard coded primitive infrastructures is the first step of infrastructure-centered model (ICM). Brokers for these four protocols are given in Figure 1 with the naming convention we used in our code. Many IoT infrastructures are available in the market by top vendors ARM, Intel (IntelIoT), CISCO (Cisco-IoE), Telit (Telit-IoT), Jasper (Jasper-IoT), Cumulocity (IoT), Davra Networks, Samsung, Qualo command HP with various features. The proposed model is shown in Figure 2. In order to utilize any infrastructure, first the information model of real infrastructures (e.g. Smart Energy, Smart Industry, Smart Government, Smart Academia, Smart Health) has to be designed using database engines.
Secondly, a generic application-programming interface (API) for a given IoT architecture is available by top IoT vendors to communicate with a particular database management system. The applicationprogramming interface API customizes the entire IoT infrastructure with respect to real infrastructures. In this paper, we have used the datasheets approach to populate our database using the datasets of a given infrastructure. The hardware architecture is static as per things in a particular infrastructure. The sensor cards are mentioned in Table 1 and parameters will remain static or fixed as defined in the datasheet of a particular object of class in the infrastructure, e.g., an object truck's datasheet will define which variables have to be measured by which sensors. The datasheets have to be provided by the regulatory authority for a given infrastructure sector, structure, actors, solution and service. In this model, analytics is centralized where the API and information model are key parameters. In this work, we have used a top-down design with Water-Fall Software Development Life Cycle (SDLC) approach. ${ }^{16}$ To ease adaptation and implementation of the system along with enhanced scalability the documentation is integrated. This model scales the best capabilities of original equipment manufacturer (OEM) $\mathrm{IoTs}^{6}{ }^{6} \mathrm{OEM}$ architectures and interoperability of IoT platforms. ${ }^{7}$ The first challenge is integration of interoperable hardware architectures and platforms. At a time, we will be dealing with one architecture using different platforms. Every proprietary IoT framework has its own definitions to classify sensors nodes, cables, drivers, network switches, IoT gateways, computers, servers and all the architecture specific software and hardware of a single vendor. ${ }^{8}$ Specifically Programmable Logic Controller(PLC), Process Automation Controller(PAC) and Distributed Control System (DCS) based nodes OPC (Object Linking and Embedding (OLE) for Process Control) is used for scalability and flexibility of merging IIoT and IoT as we have an infrastructure of Industry 4.0 (Smart Industry) also. At gateway or in parallel to broker layer we have used OPC DX (Data Exchange) and as whole middleware we have used OPC UA (Unified Architecture).

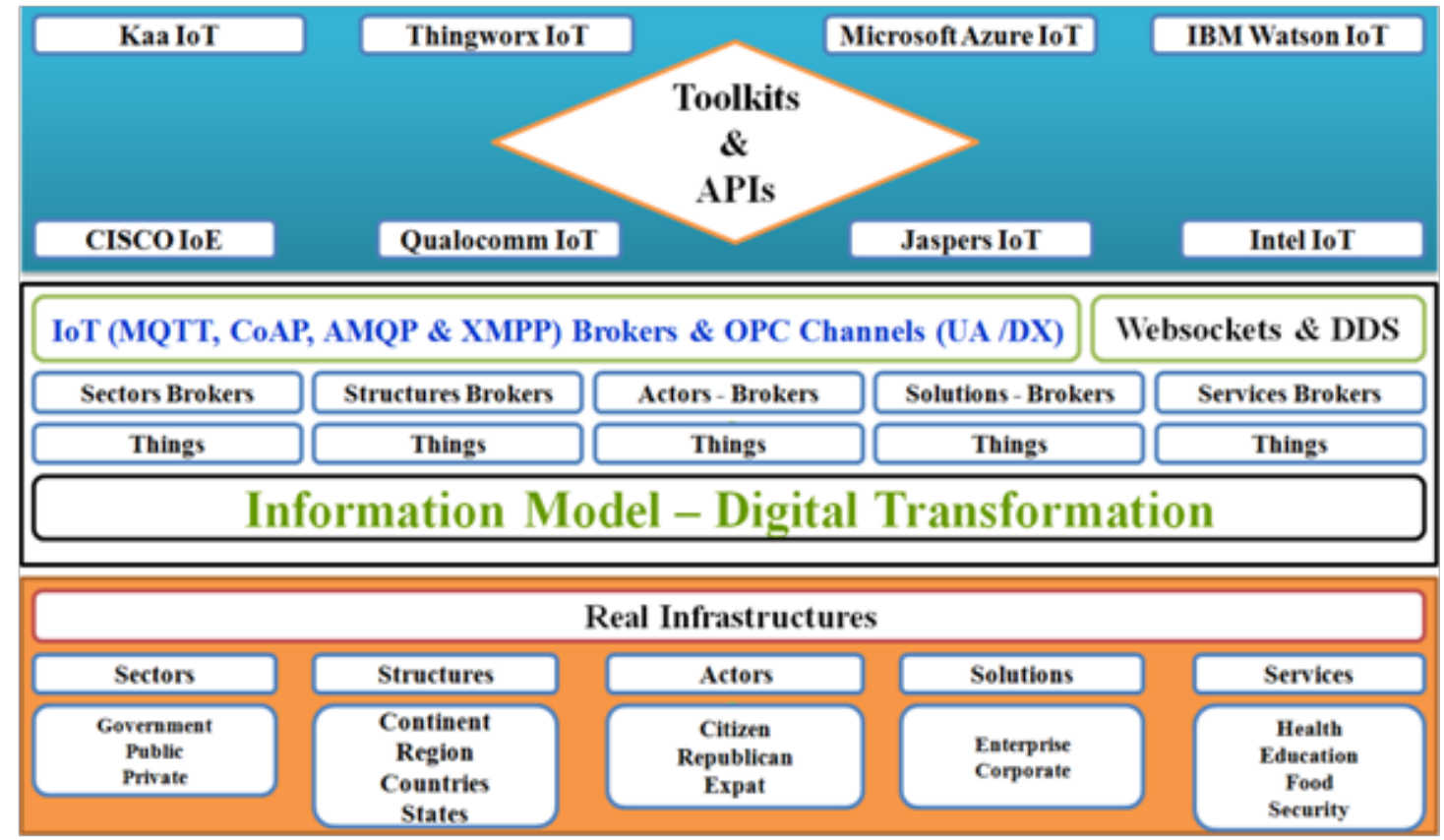

Figure 2 Infrastructure-Centered Models (ICM).

\section{Agent-centered model (ACM)}

The approach is based using the features of three giants of IoE i.e. Web of Things (WoT), Cloud Virtualization (for Virtual Desktop
Interface (VDI)) and Social Web of Things (Facebook, twitter and Instagrametc.). Cloud virtualization is one of the techniques to ensure scalability of hardware resources virtualization has to be utilized for VDIs. A normal person in this 21 st century world has only three 
personal sources of information i.e. SMS, email and social media. Excellent sources of population attention and involvement are social media and websites. Our system publishes on Facebook, twitter and Instagram. A conceptual and implemented model of our IoE is given in Figure 3. Cloud is used as infrastructure as a service (IaaS) to achieve scalability of workflow and management. Thick, thin and zero clients are the most powerful tools for utilizing scalability of hardware at lowest costs. A brief understating of thick, thin and zero clients is given in Figure 4. First of all the sensors or data acquisition points or variables will have impact factors or roles for a particular applied agent or object (e.g. Smart Grid and Smart Academia).
Then, every infrastructure set or class of agents will have its specific protocols depending on the data nature and bandwidth requirement (e.g. MODBUS, CANBUS, SNMP, PROFINET, and BACnet). In this context, every network address will be enough to identify the infrastructure being addressed. This serves two-fold purpose:

a. independence of design and implementation of the architecture and

b. Increase flexibility of platform. Every agent will have its marshalling (I/O Standardized Clusters) for remote I/Os.

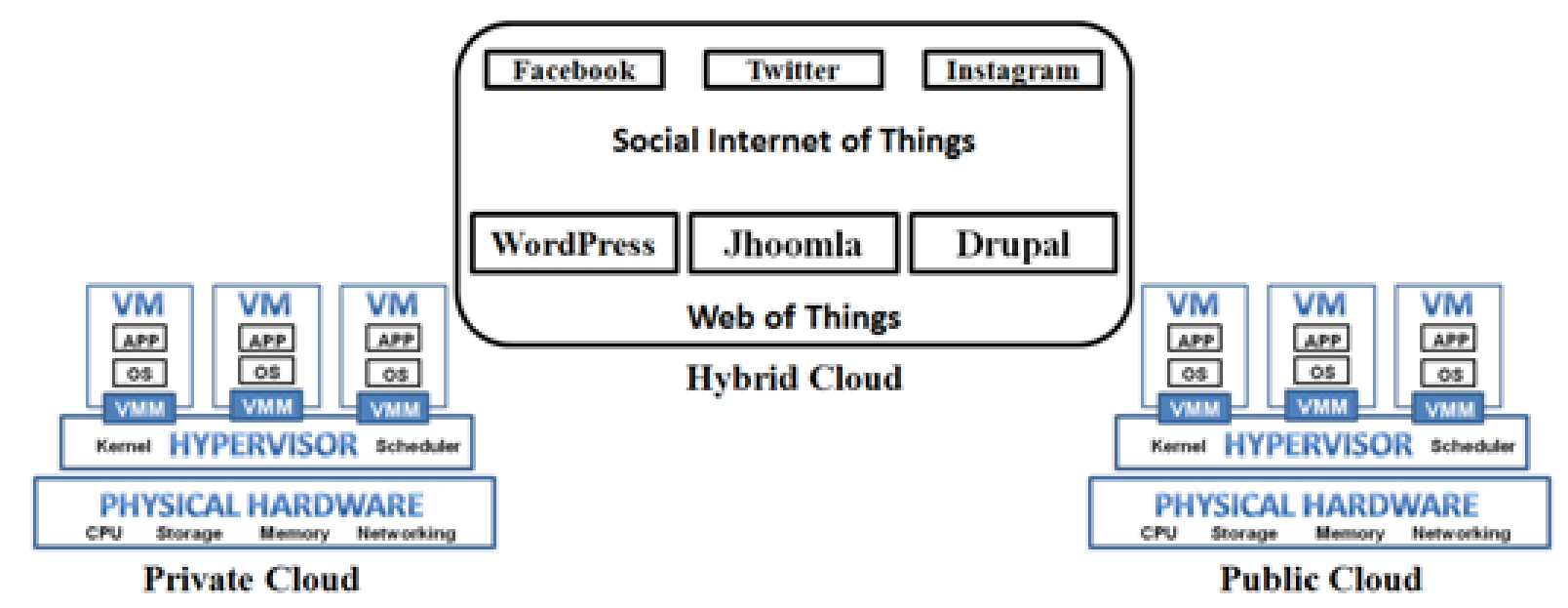

Figure 3 Hybrid Cloud and Web of Things.

\begin{tabular}{|c|c|c|c|}
\hline & Thick Clients & Thin Clients & Zero Clients \\
\hline Definition & $\begin{array}{l}\text { PCs with Operating System and } \\
\text { Clients Software }\end{array}$ & $\begin{array}{l}\text { Stations for Virtual Deskikops } \\
\text { hosted in Data Center/ / Server. }\end{array}$ & $\begin{array}{l}\text { Statious for Virtual Desiktops hosted in } \\
\text { Data Center / Sevver. }\end{array}$ \\
\hline Life Span & 4 years & 7 years & 10 years \\
\hline Hardware & A Complete PC & Atom PCs (at max) & (No Processor, No Hard disk, No RAM) \\
\hline Set tp Time & 2 bours (Average) & 5 minutes (Average) & 5 minutes (Average) \\
\hline Operating Systems & Web Brewser of Windows os & Windows or Linuax OS & No os of Application \\
\hline Security Level & 1096 & 4096 & 10046 \\
\hline Ease of Management & $10 \%$ & $50 \% 6$ & $100 \%$ \\
\hline Power Cossumption & $300 \mathrm{w}+$ & $80 \mathrm{~W}+$ & $30 \mathrm{w}+$ \\
\hline $\begin{array}{l}\text { Level of Maintenance } \\
\text { Required }\end{array}$ & 4096 & $5 \%$ & 046 \\
\hline $\begin{array}{c}\text { Bandwidth } \\
\text { Requiremeat }\end{array}$ & $<100 \mathrm{Mpts}$ & <soMpbs & SsoMpbs \\
\hline Latency & $150 \mathrm{~ms}+$ & $300 \mathrm{~ms}+$ & $125 \mathrm{~ms}+$ \\
\hline Application & & Itiple Displays, oS and level of & 4 neents \\
\hline
\end{tabular}

Figure 4 Scalability (Thick, Thin and Zero Clients).

In the proposed design, we used IP68 junction boxes and UL508 certified panels with IPC610A approved PCBs or embedded systems for commercial grade. It is based on private cloud based approach with various data protocols (e.g. MQTT, CoAP, AMQP, Web sockets and Node). It is a modular approach, which uses agents' diversification of architectures to scale up the specified infrastructure. This IoT solution is more M2Mcommunication using platform sets like Thing Speak, Kaa, AWS IoT, and Azure IoT. This model is more ubiquitous and device-safety centric. Here, the agents will be distributed as per population of citizens and concerned subjects all over the globe. A pooling mechanism will be used on the basis of fittest-to-include and then keep continuously updating data to the cloud using relevant authentications and protocols. Then all the agents connected to the ADA (Agent Data Acquisition) bridge will keep processing the data. This concept is more suitable for highly populated countries where we have health city, education city, sports city and diplomatic city, in other works, where all the infrastructures are divided in to their relevant areas as per specification. 


\section{Information model and physical system}

\section{Information model and datasheet design and development}

Every real-time system has to be designed based on real-world ergonomics. Every living and non-living thing useable or not usable has some numeric and functional description summed up as specification chart or specification sheet. Let us start with the infrastructure-centered model. The diversification of things is used as "Datasheet". The central command will have one datasheet to address; all infrastructures create the path of accessibility of resources this way. It ensures the swift transaction or movement of things moving upstream the infrastructure. It is an evolved infrastructure concept for entities like GCC, US, and EU. Simplicity of implementation ensures success. Let us apply this model for GCC; everybody will first become member of GCC, then he/she can be physically be in GCC anywhere as per requirement, instead of directly coming to Doha and start working. This suggests then that first assigns a GCC tag and proceeds to any organ like Qatar, Oman, Kuwait, Saudi Arabia UAE, or Bahrain. The true implementation of such the above union concept is a typical infrastructure-centered model as it takes all the strengths of organs and binds them into a universal skeleton with an effective handling and utilization of resources and facilitation of actors. The central portal will take the datasheet of an actor as a specified format and the actor will be placed in the system in the relevant structure, solution, or services for example. Actors have to update their datasheet instead of manual appealing. Every attribute of an actor is the arrangement or placement parameter in the infrastructure as per points. The success of this model is speed, accuracy, automation and uniformity of framework. This model will crash wherever the individual state tried to over-rule its priorities of evaluation criteria. In Figure 5, the actor code gives the lifetime exact information about its position in the entire IoT no matter what is the size of IoT; the actor is traceable. The ID is 28 characters, is a dynamic value, and is the current state as per location, structure; solution and all the attributes are defined as per the model in Figure 6. The IPV stands for IoT Priority Value, as per services or sensors used by the actor. The IPV is a dynamic value and system-generated and routed. The APV is 28 characters and stands for Appealed Priority Value, which means that the sensors or system values need to be varied as per client request. It only addresses specific cases associated with health or safety of actors and state normally stays NULL. The TT is 20 digits and stands for Transition Time, in other words it determines the duration of APV and IPV in its 10:10 digit fields. This defines how long this actor stays in this state, after which the value of IPV or APV is truncated or assigned to a new actor. The entire IoT attributes revolve around this scheme as any actor can only be at one place and in one system and structure at a particular time second. The geo-plot clearly updates based on GIS of actors. It handles all the events and infrastructure situations. Every actor has NIC sized $4 \mathrm{~mm}$ thickness color screen pager or Symbian device instead of a national id card. This Symbian device will have 5/8-portion size as battery, $2 / 8$ portion size solar panel and piezoelectric trigger circuits rest all the integrated device electronics (IDE). We implemented and tested this approach for 99,999,999 actors. Results showed that this approach is very effective in terms of efficient resource utilization, time, and cost saving per individual and aggregation. The concept used 'datasheet' is unique and has no resemblance with bio-data, $\mathrm{CV}$, resume or company profile and portfolio. It strictly deals with alphanumeric using histogram based character allocation of last 8 characters. The highest amplitude will refer to the mass population involvement and thus using the smallest ASCII value for that leading to best real-time compression. This reduces the cost of infrastructure as well as increases the density of persons per area. Example parameters are shown in packages table is shown in Table 2. Dynamic data generation and characterization leading to live dynamic compression has automatically reduced the communication bottlenecks. The MoM (Message Oriented Middleware) technique has been enhanced in our work using compression and reducing the alphanumeric character set by reducing the load on brokers and middleware at the same time. The Master Database at the IoE /IoT side has its primary keys integrated in things databases as foreign keys in D2D communication. It facilitates both scalable IoE/IoT design and implementation. OEM OPC Servers and Brokers will access individual fields and database will be updated across the hierarchy.

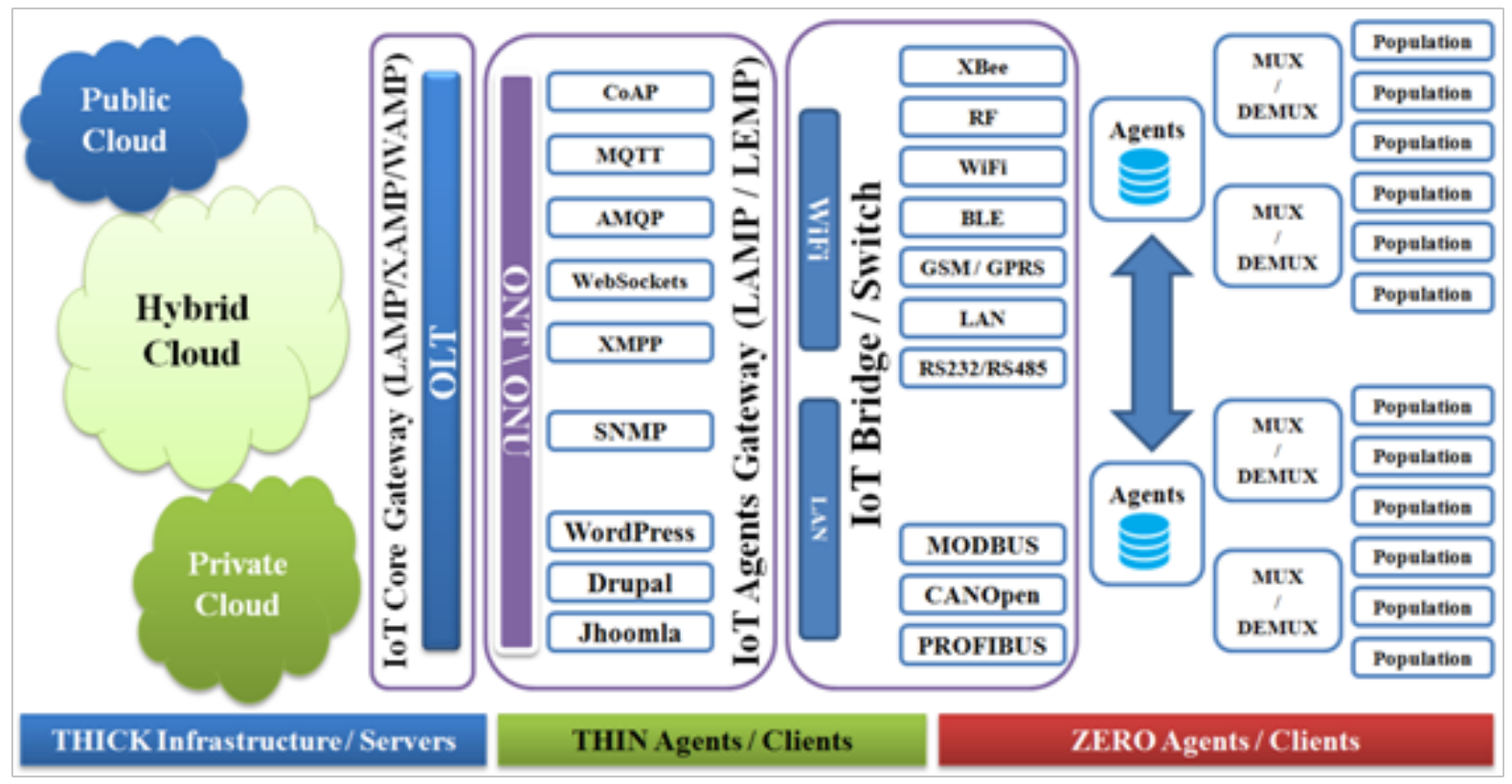

Figure 5 Agent-Centered Models. 


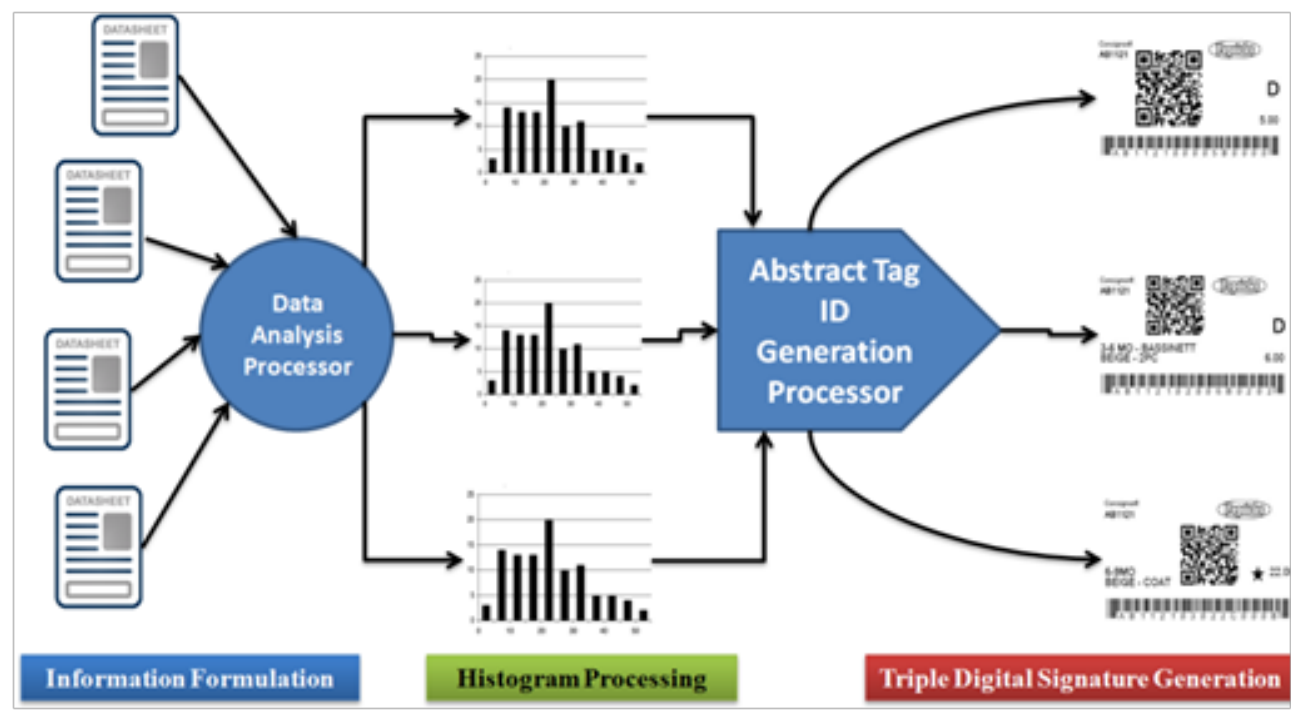

Figure 6 Abstract Tag Generation System for loT Actors.

\section{Physical system architecture and marshalling design and development}

This technique focuses on the physical presence of IoT agents in form of equidistant meshes of BLE andWiFi fogs. Assume that our cities are planned or planned in terms of homes and colonies, factories and chambers, that is, heterogeneous infrastructures where handling a population is dynamic as well as non-uniforms densities are possible due to multi-cultural environment even at un-accountable level. One DAB (Data Acquisition Box) in every home / communal unit consists of $6+$ people acquiring all the utilities and needs data. $6+$ people are the most basic social unit (2x grand-parents/guardians, $2 \mathrm{x}$ parents/guardians, $2 \mathrm{x}$ children). This DAB is stackable and incase of additional services, one need to buy an extra stacking board. Adding services is accomplished through bank payments and agents center. Water, gas, electricity, education, security, health all cards are printed upon request and penalty payment. Agent center is a small $8 \mathrm{ft} x 6 \mathrm{ft} x$ $4 \mathrm{ft}(\mathrm{LxWxH})$ cabin with automatic card printers and voucher-sticker teller machines. Just get the education card, get the sticker for new process keep on the name plate that school teacher will see, containing all the info like the subjects you paid for and hours. Put solar panels at your top of your home generate the electricity and get the payment voucher as a documentation proof and deliver it to the bank and get the cash. Card printers can print vouchers also, so only one mentioned in drawing. Agent center with zero clients is only to guide and assist in system clarification or process to be initiated or terminated. Zero client-based agents cannot correct or amend anything of population element being DA (Data Acquisition) node only. Marshalling reduces load on service centers. Service centers provide monthly and annual detailed reports plus activation of promotion services like complete user/operator manual, saving plans and rights transformation. Service centers cannot even add/remove data. Size of services center is $30 \mathrm{ft} x$ $20 \mathrm{ft} \times 15 \mathrm{ft}$ (LWH). 6 components shown in Figure 7 are fixed for all 3 packages and remaining are added as per member and utility cards purchasing.

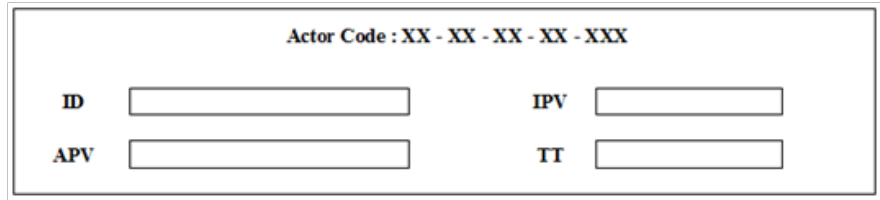

Figure 7 loT Actor:Thread and Session Structure.

\section{Results and discussion}

Package 3: Structural health monitoring has been used as a benchmark for testing ICM and ACM. Tests have produced very phenomenal results in and are shown in Figure 8 for ThingSpeak IoT platform. Sensors values from inclinometers using CAN-Open nodes have been shown as an operating example of ICM that is proprietary platform on public cloud. The system was at remote location in form of CAN-Open based tilt meters nodes connected to Raspberry Pi 3 and accessed through Wifi. The physical site chosen for this system was Qatar University Bridge as shown in Figure 9.The Wifi was linked to optical network terminal (ONT) and all nodes through ONTs forming an optical network. Optical network of ONTs was sending data to optical line terminals (OLTs). The WoT implementation can be seen in private cloud zero client screen in Figure 10. Multiple graphs, trend durations and dual cloud as shown in Figure 11, make it easier for implementation and customization for organizations and any client specific requirements (Figure 12).

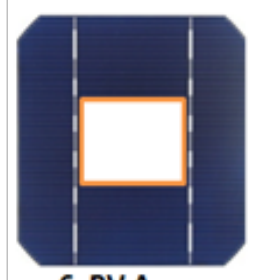

6. PV Array

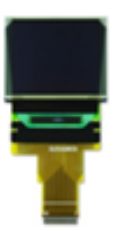

5. OLED

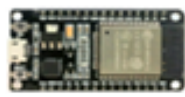

4. ESP32

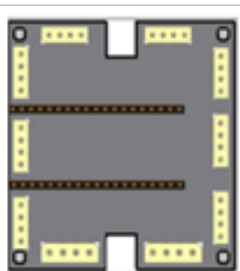

3. Sensors PCB

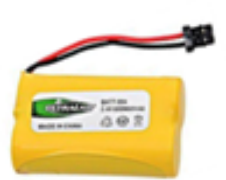

2. Battery

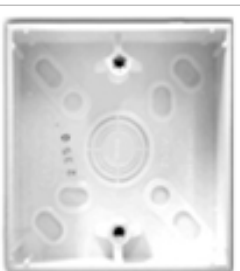

1. PVC Box

Figure 8 PDAB Components and Assembly Order (used) 


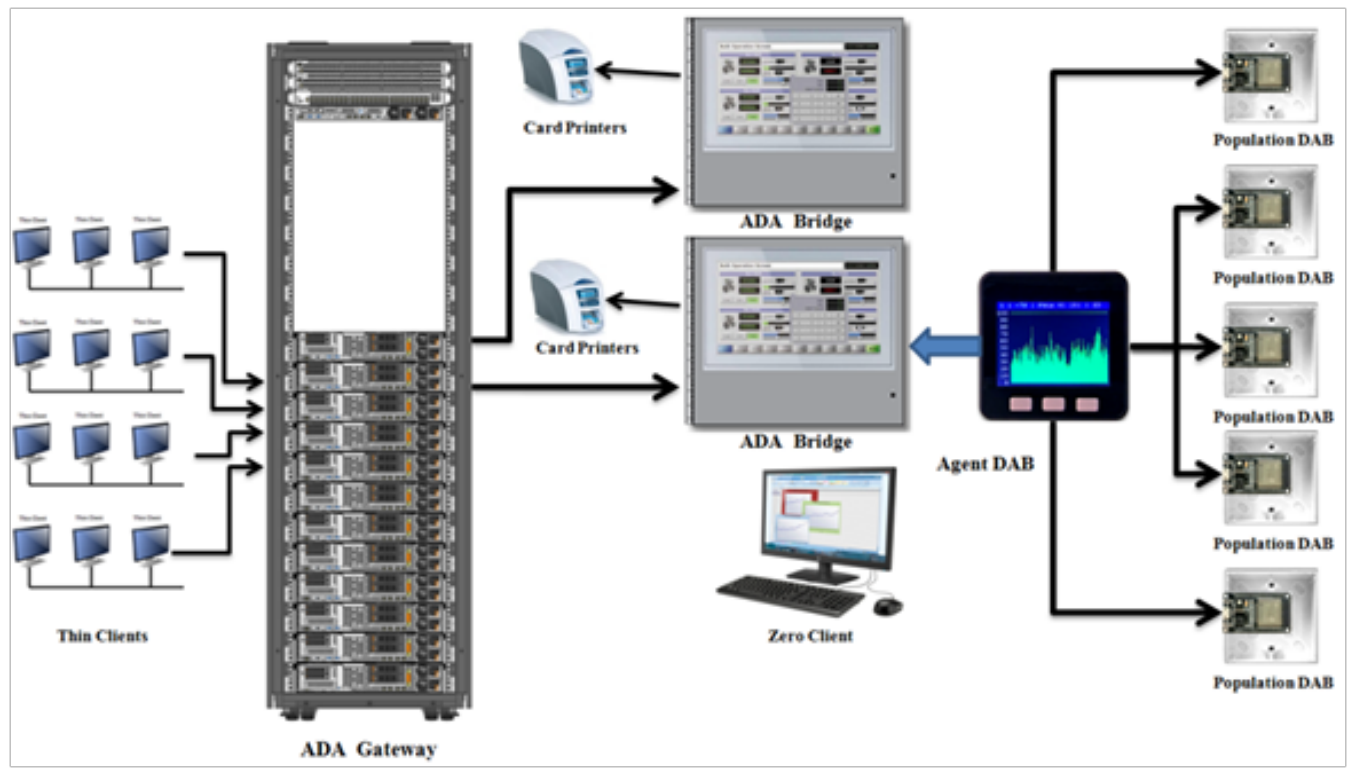

Figure 9 Ubiquitous Layers and Integrity.

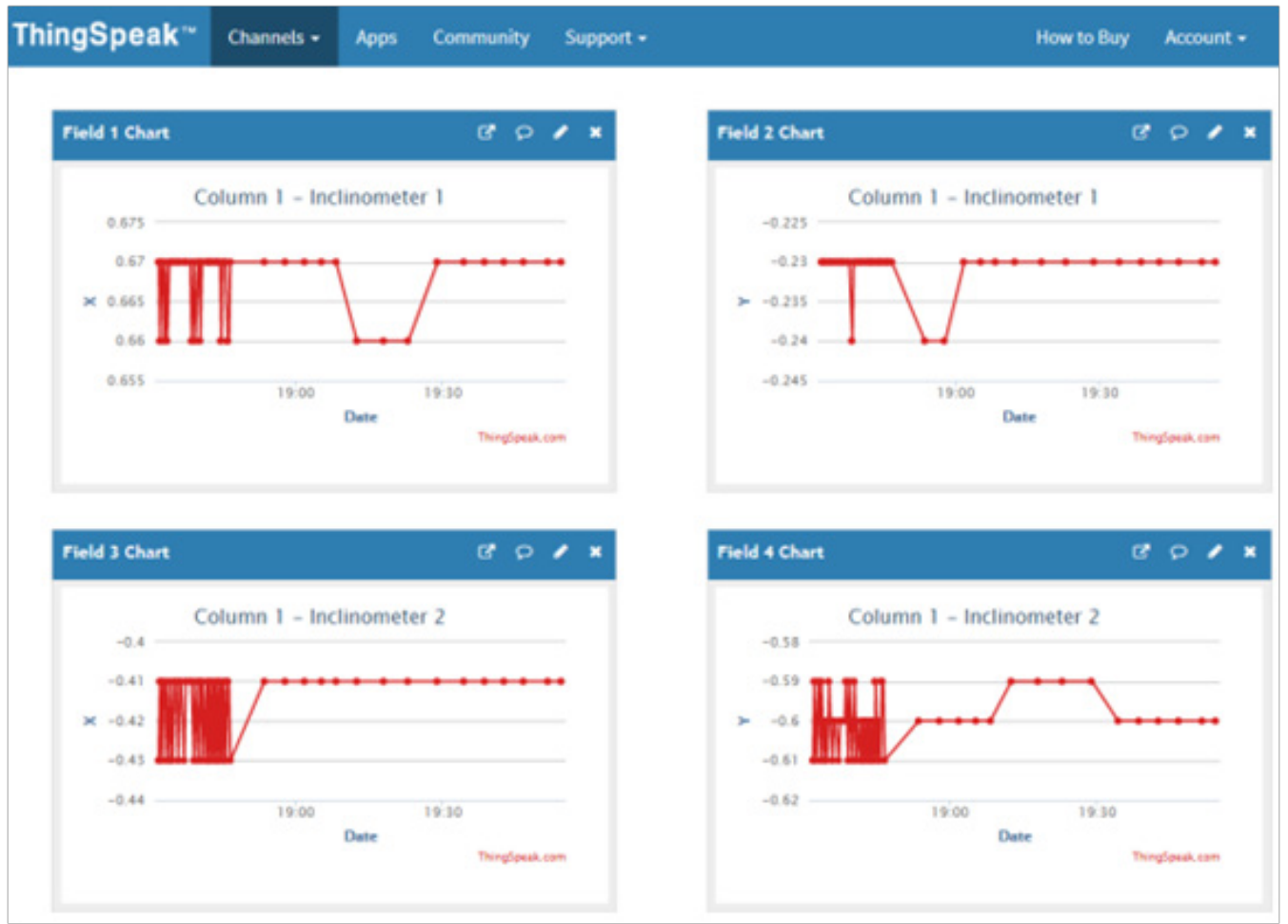

Figure 10 ICM based SHM (Package 3) Tested and Live.
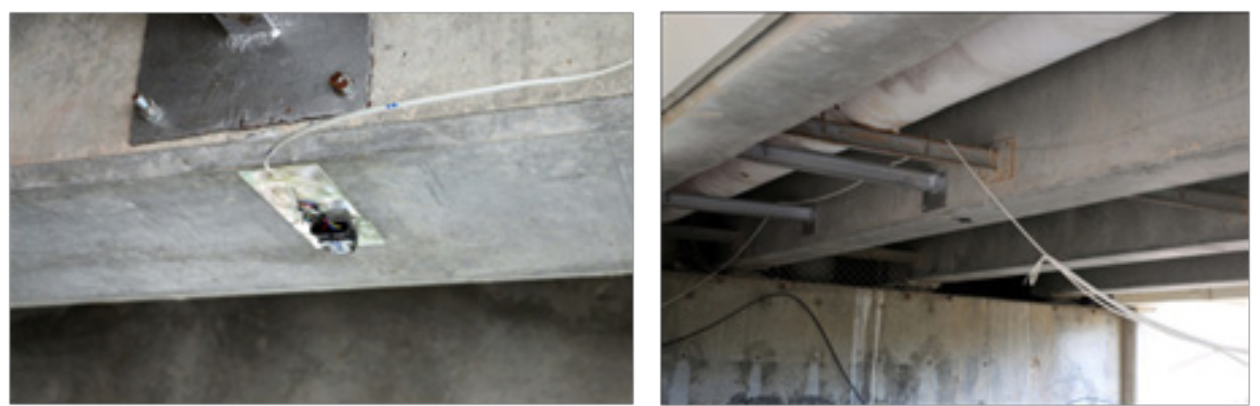

Figure I I SHM Installed as Scalable Package 3 (ACM).

Citation: Touati F, Tariq H, Al-Hitmi MA, et al. loT and loE prototype for scalable infrastructures, architectures and platforms. Int Rob Auto J. 20 I8;4(5):3 I9-327. DOI: I0.15406/iratj.2018.04.00I44 


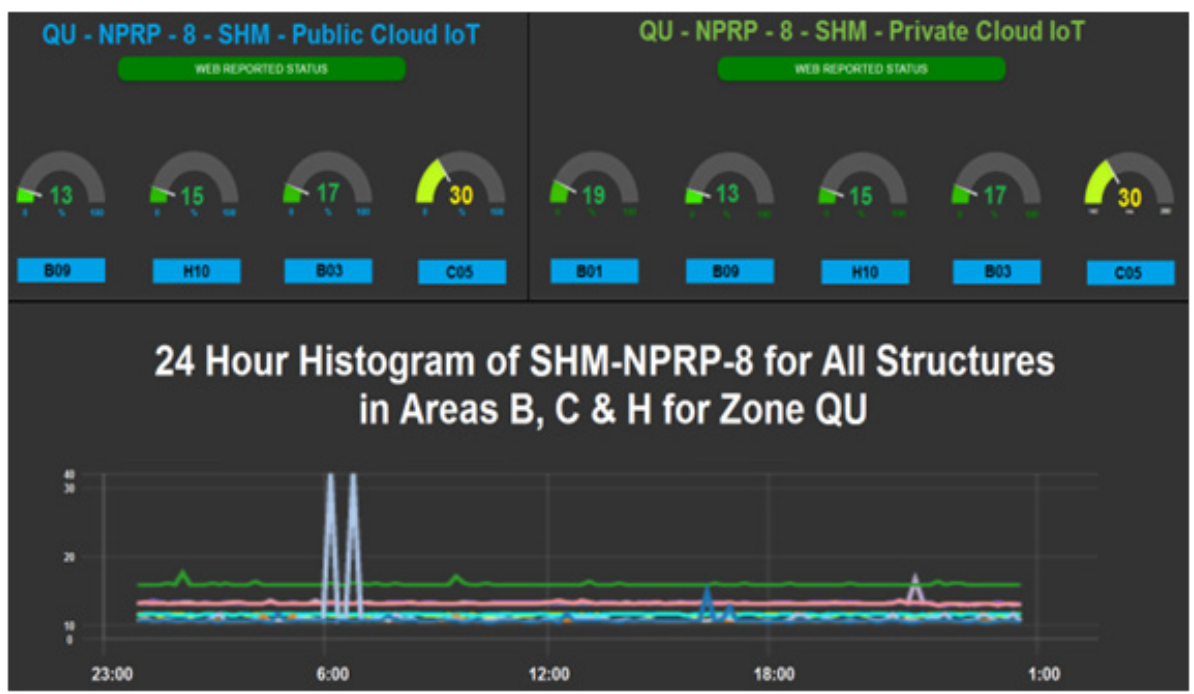

Figure I 2 SHM based Implementation for scalability and interoperability operations testing.

\section{Conclusion}

We have proposed two scalable IoT and IoE systems that have the potential of replacing and adopting the existing system integrations SCADA, OEM IoTs and PDA based systems. Multi-Broker, OPC UA/DX and Websockets based unified gateway with fiber optics communications at bottleneck can handle the massive data rate capabilities. The Symbian device proposed by us using web based (content management systems) CMS client and embedded database SQLite dealing the WoT and IoE will replace the existing personal devices du to its flexibility of being used at personal, corporate and enterprise level integrations and applications. The SCADA has been the ultimate solution for infrastructures for 4 decades and still is. Work by us also utilizes the integration capabilities of giants like Rockwell, Siemens, Schneider and Mitsubishi. Option of facility management using i.e. (Factory Talk, WinCC, Citect and third parties Indu soft, WinTr and Trace mode SCADA systems is also available in our work.

\section{Acknowledgements}

This publication was made possible by NPRP grant \# 8-17812-725 from the Qatar National Research Fund (a member of Qatar Foundation). The statements made herein are solely the responsibility of the authors.

\section{Conflict of interest}

The author declares there is no conflict of interest.

\section{References}

1. Panditab A, Jean-AnnC, James Joshua, et al. Infrastructure ecology: An Evolving Paradigm for Sustainable Urban Development. Journal of Cleaner Production. 2017;163(Suppl 1):S19-S27.

2. José Maria Cardoso da Silva, Emily Wheeler. Ecosystems as Infrastructure. Perspectives in Ecology and Conservation. 2017;15 (1):32-35

3. Jiong Jin, Marimuthu P, Jayavardhana Gubbiand SM. An information framework for creating a smart city through internet of things. IEEE. 2014;1(2):112-121.

4. Pedro Miguel Santos, JoãoGuilherme, Pereira Rodrigues, Susana B. Cruz. PortoLivingLab: an IoT-based Sensing Platform for Smart Cities. IEEE Internet of Things Journal.2018;5(2): 523-532.
5. Luca Tamburini, Maurizio Rossi, DavideBrunelli, et al. Renewable and Alternative Energy: Concepts, Methodologies, Tools, and Applications. Information Resources Management Association.

6. JasminGuth, Uwe Breitenbucher, Michael Falkenthal, et al. A detailed analysis of iot platform architectures: concepts, similarities and differences. Institute of Architecture of Application Systems. 2018:81-101.

7. Markus Hertlein, XignSys GmbH, et al. Smart authentication, identification and digital signatures as foundation for the next generation of eco-systems, and norbert pohlmann. Digital Marketplaces Unleashed. 2018:905-919.

8. RaffaeleGravina, Carlos E. Palau, Marco Manso and Giancarlo Fortino, et al. Erratum to: integration, interconnection and interoperability of IoT systems. Integration, Interconnection, and Interoperability of IoT Systems. 2017:E1-E1.

9. Bilal Afzal, Muhammad Umair M, Ghalib Shah, et al. Enabling IoT platforms for social iot applications: vision, feature mapping, and challenges. Future Generation Computer Systems. 2017.

10. XenofonFafoutis, AtisElsts, Robert Piechocki, et al. Experiences and lessons learned from making iot sensing platforms for large-scale deployments, and ian craddock. IEEE Access. 2017;(99):3140 -3148.

11. Scott Klein. IoT Solutions in Microsoft's Azure IoT Suite. 2017.

12. Ahmed Banafa. The internet of everything. 2014.

13. Ruwantissa Abeyratne. The internet of everything. Megatrends and Air Transport. 2017.

14. NitinNaik, Paul Jenkins, David Newelliew. Choice of suitable Identity and Access Management standards for mobile computing and communication. 24th International Conference on Telecommunications; 2017.

15. NitinNaik, Paul Jenkins, Philip Davies, et al. Native web communication protocols and their effects on the performance of web services and systems. IEEE International Conference on Computer and Information Technology (CIT); 2016.

16. Dae-HyeokMun, Minh Le Dinh, Young-Woo K, et al. An assessment of internet of things protocols for resource-constrained applications. IEEE 40th Annual Computer Software and Applications Conference (COMPSAC); 2016.

17. Jorge E. Luzuriaga, Pablo Boronat, et al. A comparative evaluation of AMQP and MQTT protocols over unstable and mobile networks. Consumer Communications and Networking Conference. 2015. 
18. Peter Ruckebusch, Jo Van Damme, Eli De Poorter, et al. Dynamic reconfiguration of network protocols for constrained internet-of-things devices. Internet of Things. IoT Infrastructures.2016:269-281.

19. Alghisi D, Farid Touati D. Crescini, et al. Single and multi-source batteryless power management circuits for piezoelectric energy harvesting systems. Sensors and Actuators. 2017;264: 234-246.

20. Alghisi D, Farid Touati D. Crescini, et al. A new nano-power trigger circuit for battery-less power management electronics in energy harvesting systems. Sensors and Actuators. 2017;263: 305-316.

21. Farid Touati, AlessioGalli, Damiano Crescini, et al. Feasibility of air quality monitoring systems based on environmental energy harvesting. IEEE Instrumentation and Measurement Technology Conference; 2015:266-271.

22. Farid Touati, Claudio Legena, AlessioGalli, et al. Environmentally powered multiparametric wireless sensor node for air quality diagnostic. Sensors and Materials. 2015;27(2):177-189.
23. Farid Touati, Claudio Legena, AlessioGalli, et al. Renewable energyharvested sensor systems for air quality monitoring. 26th International Conference on Microelectronics; 2015.

24. Robert Kleinfeld, Stephan Steglich, Lukasz Radziwonowicz, et al. glue. things - a mashup platform for wiring the internet of things with the internet of services. 5th International Workshop on the Web of Things; Cambridge, MA, USA. 2014.

25. Ken J Mbuki, Benard O Osero. Cost efficient education delivery by using zero clients. A Case Study of Useful Multi-Seat Platform. 2014

26. Weitao Xu, Dongfeng Yuan, Liangfei Xue. Design and implementation of intelligent community system based on thin client. and Cloud Computing. International Journal of Ad hoc, Sensor \& Ubiquitous Computing. 2014;5(4).

27. AleksandrFarseev and Tat-Seng Chua. TweetFit: fusing multiple social media and sensor data for wellness profile learning. AAAI Conference on Artificial Intelligence. 2017. 